\title{
Preparatory object rotation as a human-inspired grasping strategy
}

\author{
Lillian Y. Chang, Garth J. Zeglin, and Nancy S. Pollard \\ The Robotics Institute, Carnegie Mellon University, Pittsburgh, Pennsylvania 15213, USA \\ $\{$ lillianc, garthz,nsp\}@cs.cmu.edu
}

\begin{abstract}
Humans exhibit a rich set of manipulation strategies that may be desirable to mimic in humanoid robots. This study investigates preparatory object rotation as a manipulation strategy for grasping objects from different presented orientations. First, we examine how humans use preparatory rotation as a grasping strategy for lifting heavy objects with handles. We used motion capture to record human manipulation examples of 10 participants grasping objects under different task constraints. When sliding contact of the object on the surface was permitted, participants used preparatory rotation to first adjust the object handle to a desired orientation before grasping to lift the object from the surface. Analysis of the human examples suggests that humans may use preparatory object rotation in order to reuse a particular type of grasp in a specific capture region or to decrease the joint torques required to maintain the lifting pose. Second, we designed a preparatory rotation strategy for an anthropomorphic robot manipulator as a method of extending the capture region of a specific grasp prototype. The strategy was implemented as a sequence of two open-loop actions mimicking the human motion: a preparatory rotation action followed by a grasping action. The grasping action alone can only successfully lift the object from a 45-degree region of initial orientations (4 of 24 tested conditions) Our empirical evaluation of the robot preparatory rotation shows that even using a simple open-loop rotation action enables the reuse of the grasping action for a 360-degree capture region of initial object orientations ( 24 of 24 tested conditions).
\end{abstract}

\section{INTRODUCTION}

Robotic systems have yet to match humans in dexterity for general tool acquisition and manipulation. While robotic manipulators can be programmed to grasp objects under structured conditions, humans can easily adapt their manipulation strategies to novel task conditions.

Humans typically use a few prototypical reaching and grasping actions to pick up objects. In daily life, humans must grasp objects from a variety of initial configurations, including many which are not well-matched to familiar grasps. In our observation of human grasping, we notice that humans seldom grasp an object directly from its exact presented configuration. Instead, humans often manipulate the object to adjust its configuration prior to grasping. For example, a person might drag a mug on a table closer to the body by pulling on the handle with non-prehensile contact. In another scenario, to grasp a pen from a table surface, the fingertips may be used to quickly pivot the handle to orient the tip for writing.

These are examples of what we refer to as "preparatory manipulation." Preparatory manipulation occurs whenever the interaction first adjusts the object configuration on the supporting surface prior to the final grasp (Fig. 1). This approach takes advantage of the object's movability on the surface to effectively change the intermediate task parameters. In such

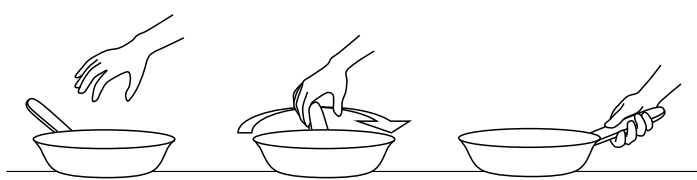

Fig. 1. Preparatory manipulation adjusts the object configuration in the workspace prior to grasping. One example of preparatory manipulation is preparatory object rotation, shown here for a handled pan, where the object orientation is adjusted before grasping.

cases, the action in anticipation of a grasping task includes changes in the object configuration in the environment prior to grasping, in addition to the manipulator reaching movement and hand pre-shaping.

In this paper, we examine one type of preparatory motion as a manipulation strategy used by humans: rotating a handled object using a pushing contact prior to a lifting grasp. We show that the preparatory rotation of an object allows for a single grasp action to be reused for a much wider range of object configurations. We first study the preparatory rotation strategy used by humans. Then we implement the strategy on an anthropomorphic robot manipulator to investigate how preparatory rotation can extend the effective workspace of a well-tuned grasping action.

\section{RELATED WORK}

This work utilizes a simple pushing strategy to rotate an object prior to grasping it. The benefits of utilizing the support surface as a passive manipulator to increase workspace and load limits are familar from the push-planning literature [1]. Much of that work is concerned with the automatic planning of push manipulations to reorient objects on a supporting surface with either known or uncertain contact conditions. For example, Lynch and Mason [2] explore the conditions for complete control of a rigid sliding object while pushing to an arbitrary pose on a plane. In contrast, our emphasis is on the identification of specific heuristic action sequences which humans choose in response to prototypical situations. Thus, rather than modeling the general push dynamics, we have deliberately chosen an example with simple contact conditions and high error tolerance which admits a trivial ad-hoc solution to the push-planning problem.

Planning methods for pushing manipulation have been demonstrated on other humanoid platforms in recent work [35]. Our focus is on the benefits of using pushing to reuse grasping strategies for a wide range of object orientations, rather than on the planning of the pushing action alone. 
The strategy is also a simple example of sensorless manipulation. It does not measure object state, but assumes the object lies within a bounded set of initial configurations. It also uses a contact strategy chosen to reduce the uncertainty of the object state until it lies within the much smaller set of graspable states. Again, this type of strategy has been wellstudied in the motion planning literature [6], but our emphasis is on identifying small sets of primitives which can be used to quickly construct adequate solutions to typical grasping problems rather than general optimal planning.

Related literature also include studies of human manipulation from the psychology and motor control community. Rosenbaum and colleagues have investigated the selection of hand grips for a variety of handle transport and handle rotation tasks where a cylinder is grasped and then placed at different goal configurations $[7,8]$. This has led to several inquiries testing how perceived end-state comfort of a task affects the choice of initial hand grasps in object transport tasks $[9,10]$. In recent work, Rosenbaum and Gaydos [11] present a relative cost approach for evaluating the movement costs for tasks such as object-positioning and object-rotation.

Our experiments are the first, to our knowledge, to explore what we refer to as preparatory manipulation strategies in human subjects. Departing from the previous studies [8, 12] on manipulation of lightweight objects, we chose to investigate human lifting of heavy objects since we believe preparatory manipulation is most relevant to more demanding tasks.

\section{PREPARATORY ROTATION IN HUMANS}

The aims of the human study are to quantify the consistency of object rotation as a preparatory strategy and to examine possible criteria that the strategy may optimize. In this section we review the basic experimental protocol and major findings. A complete, detailed description can be found in Chang and Pollard [13].

\section{A. Experimental procedure}

Ten right-handed adults (5 male, 5 female) volunteered for the study (age $=26.7 \pm 3.5$ years [mean \pm standard deviation], height $=1.67 \pm 0.09 \mathrm{~m}$, weight $=58.7 \pm 10.9 \mathrm{~kg}$ ) All participants signed informed consent forms approved by the Institutional Review Board.

Participants performed the object lifting tasks in a kitchen counter top setting (Fig. 2). The object start position was located on the right side counter area. The object goal area was located $0.8 \mathrm{~m}$ to the left of the start position and was marked by a circular cover over the bottom left stove burner. At the start position, the object was presented in one of eight possible orientations, indicated by the direction of the object handle. In orientation 1, the handle directly faces the participant (Fig. $2 b)$. The two handled objects tested for all ten participants were a plastic water jug and a cooking pan, both without lids (Fig. 3). The objects were filled with water for a total mass of $3.4 \mathrm{~kg}$ for the jug and $1.5 \mathrm{~kg}$ for the pan.

Kinematic data for the participant and objects were recorded at $120 \mathrm{~Hz}$ using a Vicon camera system (Vicon Motion

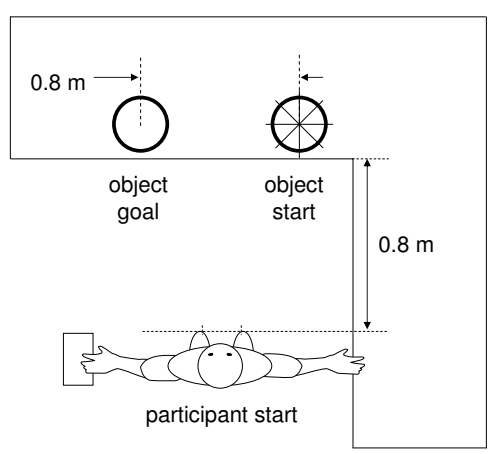

a

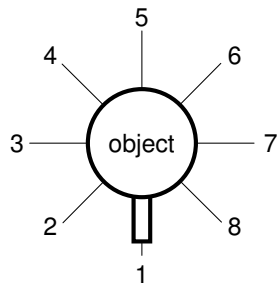

Fig. 2. General layout of the experimental setting. (a) Participants started in a standing position facing the countertop setting. Participants transported the handled objects from the start position to the goal position with their right hand. (b) In each trial, the handled object started in one of eight orientations defined by the handle direction. In the figure, the handled object is in orientation 1, where the handle is facing the participant.

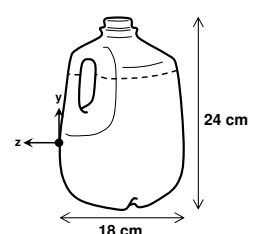

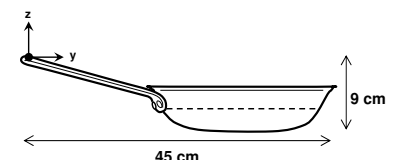

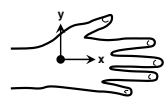

Fig. 3. Participants transported two different handled objects filled with water, (a) an uncapped water jug and (b) a cooking pan. The dashed line indicates the approximate water level. The coordinate systems located at the object handles provide a reference frame for measuring the (c) hand dorsum configuration during the object grasp.

Systems, Los Angeles, California, USA). Motion of the full body, including hands and fingers, was tracked by 80 reflective markers attached to the participant [13].

In each lifting task trial, the participant started facing the counter at a distance of $0.8 \mathrm{~m}$ from the counter edge, such that the object was outside of arm's reach. For all trials, the task was to move the object to the goal position without spilling any water. No specific object orientation was required at the goal. Participants were instructed to perform the transport task at a self-selected speed with no time constraints.

The experiment consisted of three phases to examine three types of task scenarios (Fig. 4). The first phase served as practice trials to familiarize the participants with the task setting. Participants were instructed to complete the transport task with no restrictions, using either or both hands as desired.

The second phase (Fig. 4b) investigated unimanual lifting performance in response to the different object handle orientations. The purpose of this phase, as the main portion of the experiment, was to observe to what extent object preparatory adjustment would be used as a strategy to compensate for changes in object orientation. Participants were instructed to complete the transport task using only their right hand to contact the object. Besides this unimanual constraint, there were no restrictions on the task performance. The verbal 


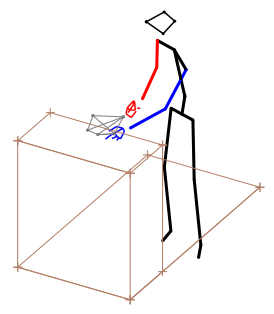

a

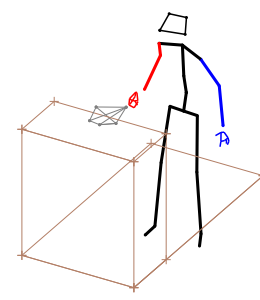

b

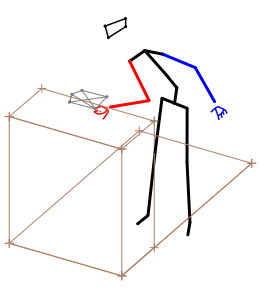

$\mathrm{c}$
Fig. 4. Participants lifted objects in three task scenarios. (a) Unconstrained practice trials where bimanual manipulation was permitted. (b) Unimanual constraint trials where only the right hand was permitted to contact the object. (c) Object motion constraint trials where sliding contact of the object on the surface was not permitted.

instructions did not suggest preparatory rotation or sliding motion as a strategy, as it was our intent to observe what strategies the participants would naturally select.

The final phase (Fig. 4c) tested how participants would respond to different object handle orientations in the absence of preparatory object adjustment on the counter surface. The task performances from this third phase provide a reference measurement for analyzing the object adjustment motion in the second phase. Participants were instructed to transport the object using only the right hand and without sliding motion on the surface prior to lifting the object.

\section{B. Motion data analysis}

The key time point of object lift-off from the surface is the focus of our data analysis. This time point was estimated automatically from the trial data as the time frame when the object's vertical motion exceeded $1 \mathrm{~cm}$. Four metrics (Table I) were computed from the participant's body pose at the lift-off time frame: object rotation, joint torque load, grasp orientation, and grasp location (described in detail in Chang and Pollard [13]).

Object rotation was measured as the difference between the initial object orientation and the object orientation in the liftoff frame. We computed the absolute amount of rotation so that there was no distinction between clockwise or counterclockwise rotation.

Upper body joint torques were estimated from the lift-off body pose using segment mass ratios and center of mass locations from anthropometry [14]. Given the fitted joint center locations for the lower back, shoulder, elbow, and wrist, joint torques were calculated from the loads due to distal limb segment weight and the object weight. The four joint torques were combined into a single metric as the sum of squared joint torques.

The configuration of the hand dorsum coordinate system was then computed with respect to the reference coordinate system attached to the object handle (Fig. 3). The grasp orientation was measured as the angle magnitude of the single axis-angle rotation which would align the hand dorsum coordinate system to the object handle coordinate system. The grasp location was measured as the distance between the hand jug lifts with unimanual constraint pan lifts with unimanual constraint
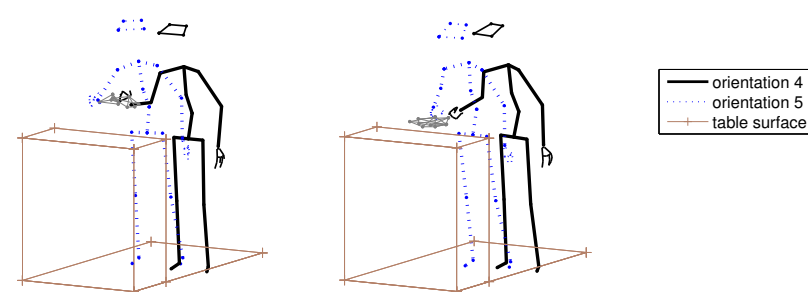

jug lifts with object motion constraint pan lifts with object motion constraint
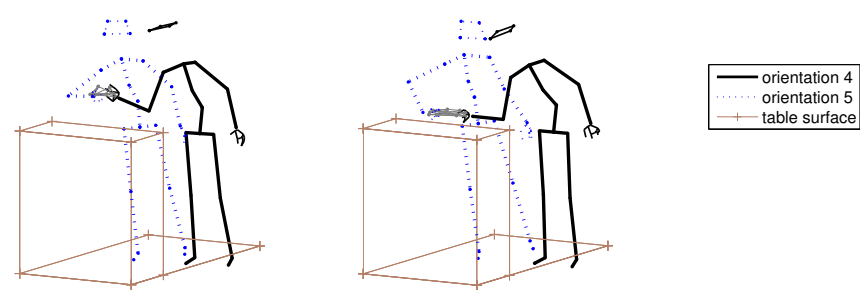

b

Fig. 5. Visualization of the body postures at object lift-off for a sample subject. Poses are shown for the trials with initial object orientation 4 and 5, where the handle faced away from the participant. (a) Poses for the trials with the unimanual constraint. (b) Poses for the trials with the additional object motion constraint. The lift-off poses for the unimanual constraint trials were more similar to each other because the object motion adjusted the handle direction toward the participant. When object motion was not permitted, the lift-off poses are more varied. In the most extreme cases for initial object orientation 4 and 5 , the torso is tilted over the countertop and the elbow is held away from the side of the body.

coordinate system origin at the proximal end of the third metacarpal and the object coordinate system origin at the base of the handle (Fig. 3).

The overall set of dependent variables examined in this study (Table I) were the metric differences between the unimanual constraint trials (second phase) and reference object motion constraint trials (third phase). The differences in metrics were computed between matched pairs of trials performed by the same participant on the same object for the same initial handle configuration, with only a difference in the task constraint. The differences were computed for the two sets (repetitions) of 8 trials per object in the unimanual constraint phase with respect to the single set of 8 trials per object in the motion constraint phase. We analyzed the difference metrics with linear mixed-effect (LME) models [15] (see [13] for details).

\section{Human study results}

When participants were only restricted by the unimanual constraint, they often rotated the object on the countertop surface to a new orientation before lifting and transporting the object to the goal. The resulting body poses at object lift-off (Fig. 5) were similar in terms of the upright torso orientation and object handle directed toward the participant. In contrast, when the object rotation strategy was precluded by the object motion constraint, the resulting body poses at 
TABLE I

RESPONSE METRICS OBSERVED FOR EACH TRIAL WHERE THE PARTICIPANT LIFTED AND TRANSPORTED A HANDLED OBJECT FROM THE PRESENTED OBJECT START ORIENTATION. THE DATA ANALYSIS MODELED THE DIFFERENCES IN EACH OF THE METRICS BETWEEN THE UNIMANUAL CONSTRAINT TRIALS AND OBJECT MOTION CONSTRAINT TRIALS.

\begin{tabular}{ll}
\hline Object lift-off posture metric & Computation notes \\
\hline Object rotation & Absolute difference between lift-off handle angle and initial handle angle \\
Joint torque load & Sum of squared torques over torso, shoulder, elbow, and wrist \\
Grasp orientation & Angle of single rotation between hand frame and object frame \\
Grasp location & Distance between origins of hand frame and object frame \\
\hline
\end{tabular}

object lift-off were more varied in the torso orientation and arm configuration. For trials where the object handle faced away from the participant, the torso was often tilted over the countertop surface with the elbow extended away from body to achieve the grasp of the object handle. One participant chose to abort one lifting trial in the object motion constraint phase after grasping and attempting to lift the pan from handle orientation 5 without object motion along the surface.

Under the unimanual constraint (second phase), the amount and direction of object rotation varied depending on the initial handle orientation, as did the object orientation at the lift-off time frame (Fig. 6). In general, the selected lift-off orientations were clustered in a capture region centered around handle orientations 1 and 8 , on the participant's right side. Thus, the scale for the handle orientation variable is centered at the midpoint angle between orientations 1 and 8 for the statistical analysis [13].

The differences in object rotation (Fig. 7) between the unimanual constraint trials and object motion constraint trials were the largest for initial handle orientations 4 and 5, which are opposite the capture region at orientations 1 and 8 . The LME analysis found the quadratic trend of rotation amount vs. initial angle to be statistically significant $(p<0.00005)$ [13].

The difference in the sum of squared joint torques also exhibited a significant quadratic trend with initial handle orientation $(p<0.00005)$ (Fig. 8). The joint torque metric for the object motion constraint trials was greater than those for the unimanual constraint trials, as seen from the primarily nonnegative differences (Fig. 8). We tested the linear correlation between the difference in object rotation and the difference in the joint torque metric using the Pearson correlation coefficient. The correlation was statistically significant $(\alpha=.05)$ for 8 of the 10 participants for the jug lifts and for all 10 participants for the pan lifts.

Hand grasp configuration differences (Fig. 9) also increased when the initial handle directions faced away from the participant. There was a significant quadratic trend for the grasp orientation difference $(p=0.0015)$. For the grasp orientation, there is also a significant linear orientation effect $(p<0.00005)$ which is seen in the asymmetry of the mean regression curve (Fig. 9b). The asymmetry is due to the unimanual constraint of right-hand lifting. The grasp differences are smaller for handle orientations 5 to 8 , where the grasps were reached on the right hand side. Handle orientations 1 to 4 required the right hand to cross the body to reach the left
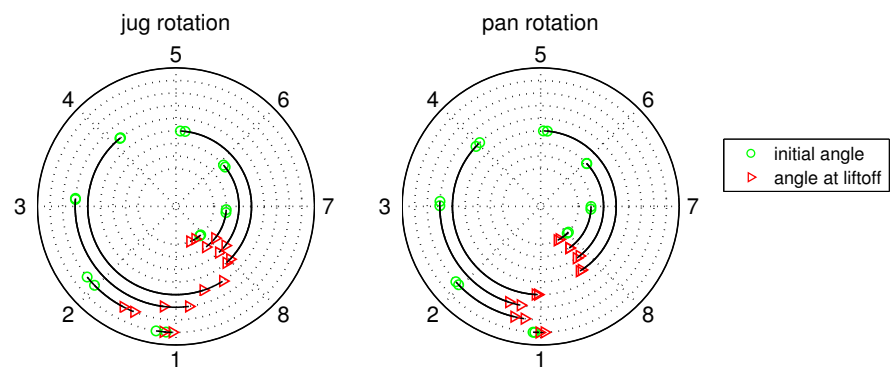

a

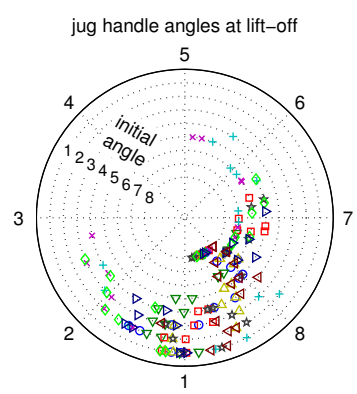

pan handle angles at lift-off

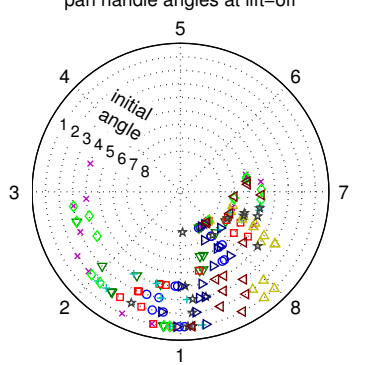

\begin{tabular}{|ll|}
\hline$\circ$ & $\mathrm{S} 1$ \\
$\nabla$ & $\mathrm{S} 2$ \\
$\square$ & $\mathrm{S} 3$ \\
+ & $\mathrm{S} 4$ \\
$\times$ & $\mathrm{S} 5$ \\
$\triangle$ & $\mathrm{S} 6$ \\
$\dot{\mathrm{S}}$ & $\mathrm{S} 7$ \\
$\otimes$ & $\mathrm{S} 8$ \\
$\triangleright$ & $\mathrm{S} 9$ \\
$\triangleleft$ & $\mathrm{S} 10$ \\
\hline
\end{tabular}

Fig. 6. Visualization of the object rotation prior to lift-off for the different initial handle orientations. (a) Initial and lift-off orientations for the unimanual constraint trials for one participant. (b) Object lift-off angles for the unimanual constraint phase for all 16 trials for all participants.
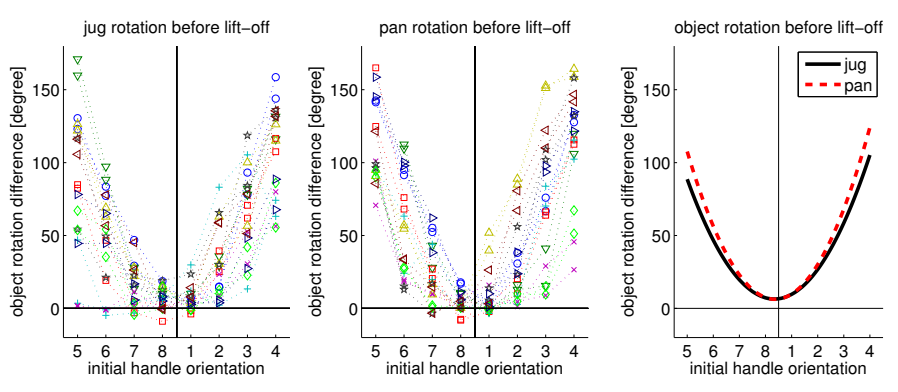

Fig. 7. Difference in object rotation versus initial handle orientation. (a) Individual participant results. (b) Mean regression curve determined from the LME regression model. The differences are the object rotation amounts in the two unimanual constraint trials minus the object rotation in the object motion constraint trial. The amount of object rotation prior to lift-off increases as the handle orientation moves further from the baseline orientation naturally preferred for the lifting task.

side for the object motion constraint trials, which led to large 

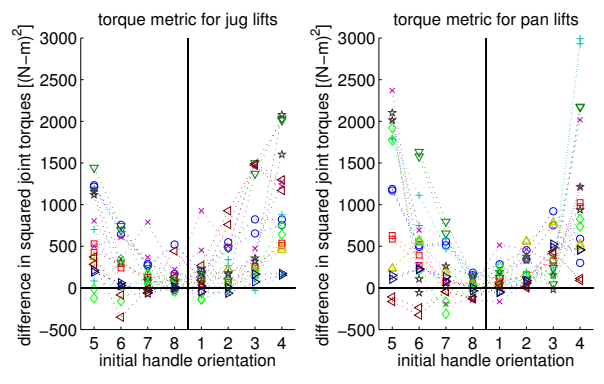

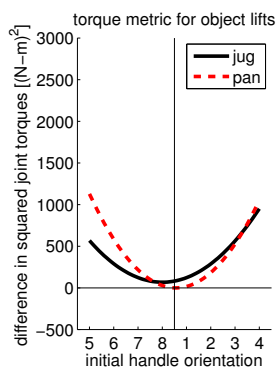

Fig. 8. Difference in the sum of squared joint torques versus initial handle orientation. (a) Individual participant results. (b) Mean regression curve determined from the LME regression model. The differences are the torque metrics in the two unimanual constraint trials subtracted from the torque metric in the object motion constraint trial. The torque metric also follows a quadratic trend, similar to that for the object rotation metric.
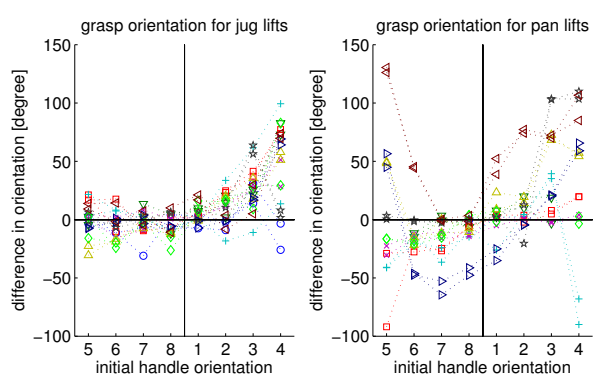

a
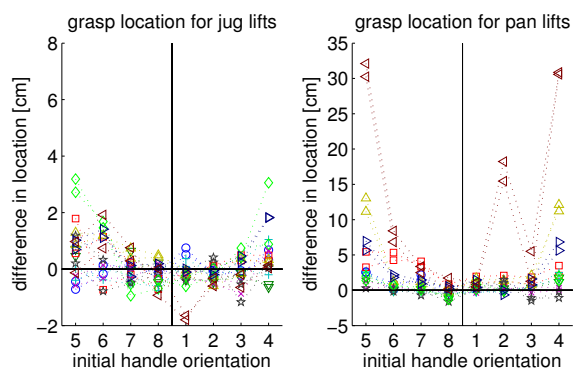

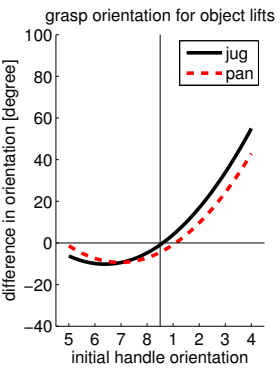

b

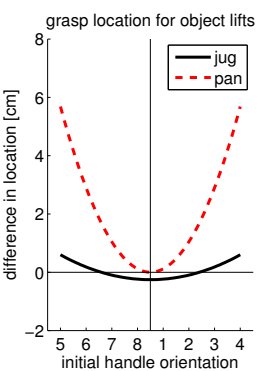

Fig. 9. Difference in grasp as represented by the hand dorsum orientation and location with respect to the object frame. The differences are the grasp metrics in the two unimanual constraint trials subtracted from the grasp metric in the unimanual and object motion constraint trial. (a) Individual participant results for grasp orientation difference. (b) Mean regression curve determined from the LME regression model for the grasp orientation difference. (c) Individual participant results for the grasp location difference. (d) Mean regression curve determined from the LME regression model for grasp location difference.

\section{differences in grasp orientation.}

Similarly, there was a significant quadratic trend for the grasp location difference $(p<0.00005)$. The quadratic curvature was higher for the pan lifts than for the jug lifts. This was due to the length of the pan handle, which allowed participants to grasp the object at several different positions. For some participants, the grasp location for lifting the pan changed dramatically in the object motion constraint phase where no sliding allowed. Instead of grasping close to the handle end as they did in the unimanual constraint phase, they lifted the pan with a grasp closer to the center of the pan when the handle was further from reach. The use of preparatory rotation strategy when it was permitted in the unimanual constraint phase might be due to the preference to grasp the object handle at position requiring less arm reach, even though other grasps were feasible when object motion on the surface was not permitted.

\section{Observations on human strategy}

Overall, we have found that the preparatory rotation of heavy objects increases with the change in handle orientation away from the preferred capture region. When participants are instructed not to pre-rotate the object prior to lift-off, they are still able to successfully complete the object transport task. However, without adjusting the object orientation prior to lifting, participants performed the lifting task with different body poses with tilted torsos and extended elbow positions in order to reach the object handle. Our results suggest that the preparatory object adjustment may be desirable because it allows the object lift to be performed with lower joint torque load in the upper body and/or with a preferred grasp of the object handle.

Our experiments investigated the preparatory object adjustment in the specific context of right-handed lifting and lateral transport across the body. We focused on the effect of the initial object orientation on the selected body posture at object lift-off, but several other factors may affect the preparatory manipulation. We would expect similar adjustment strategies in other tasks with different constraints. For example, changing the location of the goal may result in a shifted capture region for the lift-off handle orientations. Other factors include whether the right or left hand manipulates the object, timing restrictions for the task completion, object weight, and object handle geometry.

Our analysis focused on the difference in performance in terms of metrics computed from lift-off time frame, which is assumed to be a representative, quasi-static snapshot of the performance. Future work investigating preparatory manipulation could analyze the dynamics of the motor behavior over the entire trial duration.

\section{IMPLEMENTATION ON AN ANTHROPOMORPHIC ROBOT MANIPULATOR}

Inspired by the human use of preparatory rotation in lifting handled objects, we implemented the strategy on an anthropomorphic robot manipulator. The preceding results from the human subject experiments suggest that the preparatory rotation strategy may be preferred to direct grasping for multiple reasons, such as grasp reuse or decreased joint torque. Our goal for the robot implementation was to focus on how preparatory rotation can enable grasp reuse in order to extend the effective workspace of a grasping prototype. A single welltuned grasping sequence may only successfully lift a specific object from a small set of initial orientations. Preparatory rotation would reconfigure the object such that the subsequent execution of the single grasping sequence is reusable for a wider range of initial orientations. 


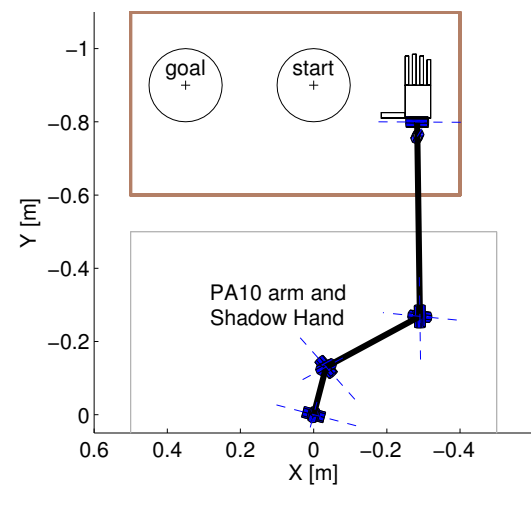

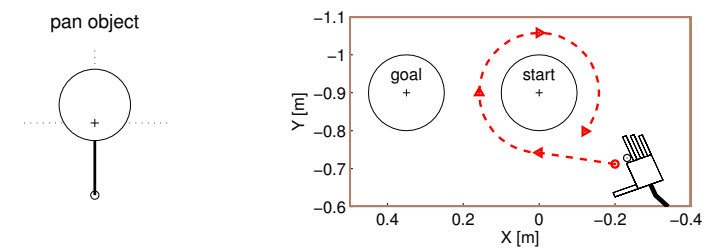

start handle angles

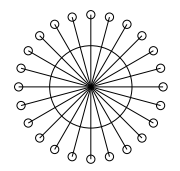

Fig. 10. Layout of the manipulator experimental setting. (a) The right-handed anthropomorphic robot arm transported a handled pan from the start position to the goal position on a table setting. (b) The pivot point of pan object is placed at the start position. The pan started in one of 24 orientations defined by the handle direction. The orientations are nominally spaced by 15 degrees

Our anthropomorphic manipulator system consists of a Mitsubishi PA-10 7-DOF manipulator with a 24-DOF Shadow Hand C3 end-effector (Shadow Robot Company, London, UK). Foam padding is attached to the Shadow Hand to modify the palm geometry. The task specified for the robot implementation mimicked the task conditions in the human studies (Fig. 10). The object starting position on the table is located $0.9 \mathrm{~m}$ in front of the manipulator base. The object goal position is located on the table $0.35 \mathrm{~m}$ to the left of the start position. As in the human studies, the object could start in one of several configurations. Twenty-four handle directions were selected to sample the full 360-degree orientation space at intervals of 15 degrees. The object in the robot experiments was a small cooking pan with a handle. The pan was empty and had a total mass of $0.46 \mathrm{~kg}$. Optical markers were attached to the pan in order to track the object configuration using the camera system described in Section III-A.

\section{A. Open loop action sequences}

The grasping strategy using preparatory rotation was implemented as two manually-programmed open-loop actions (Fig. 11). One action is the grasping action for lifting and transporting the pan by its handle (Fig. 11b). The other action is the preparatory rotation action for reconfiguring the handle orientation prior to grasping (Fig. 11a).

The grasping action was intended to mimic the underhand grasp of the pan observed in the human study trials with the right-hand unimanual constraint. The intended handle orientation for the grasping action was the direction facing toward the manipulator and slightly toward the right, as observed in the human examples (Fig. 6). The grasping action is a sequence of three motion components: an approach motion, the grasp motion, and the transport motion (Fig. 11b). In the approach motion, the Shadow Hand maintains a relaxed open-hand pose. During the grasp motion, the PA-10 arm configuration remains fixed while the hand's finger joints close around the handle. The hand then maintains a tightly-

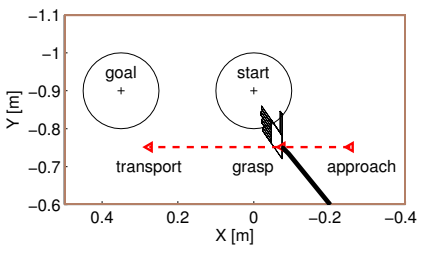

b

Fig. 11. Schematic of the two open loop actions implemented on the robot manipulator. (a) In the preparatory rotation action, the hand traces a circular arc around the starting position to rotate the pan using a single index finger contact with the edge of the handle. (b) In the grasp action, the hand moves in a relatively straight path across the table to first approach the object from the right, grasp the handle, and then transport the object left to the goal.

closed pose during the transport motion while the PA-10 arm moves to lift and transport left to the goal position. All three motion components were manually programmed for the specific object, intended handle orientation, start position, and goal position. Initial hand contact with the object often occurred during the approach motion, when the palmar side of the fingers contact the right edge of the handle.

The rotation action was implemented as a pushing motion using single-finger contact with the object to rotate the cooking pan around its natural pivot point (Fig. 11a). The index finger was flexed 90 degrees such that it pointed normal to the palm. The thumb and other three fingers were extended in the plane of the palm, which remained parallel to the table during the rotation action. The index fingertip first approaches the object start position along an initial straight segment. Then the index fingertip traces a circular arc of 315 degrees in a clockwise direction around the object start position and ends within the intended grasp capture region. Tracing the full 315 degree arc path allows the open-loop rotation action to be executed identically regardless of the initial pan handle angle. The 45 degree gap in the circular path was deliberately designed to avoid contacting the object if the handle starts within the original capture region of the grasping action alone.

\section{B. Empirical evaluation}

To measure how well the preparatory rotation enables reuse of the grasping action, we compared the grasping action alone to the sequence of the preparatory rotation followed by grasping action. The two methods were each tested on the different initial handle orientations in a set of 24 consecutive trials. The manipulation was considered successful if the grasp lifted the pan off the table surface and transported the pan to the goal position.

In the 24 consecutive trials using the grasping action alone, the manipulator successfully grasped and transported the pan to the goal position for 4 of the 24 initial handle angles (Fig. 12a.) The empirical capture region of the grasping action alone was 45 degrees. The grasp component works best for the two handle angles in the center of the capture region. For the two outer angles of the region, the handle was rotated toward the center of the region by either a clockwise push from the approach component or a counterclockwise push from the 


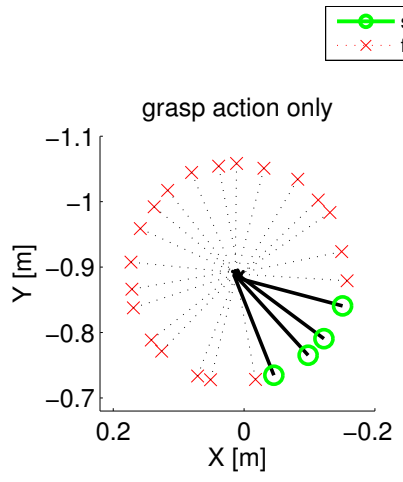

$\mathrm{a}$

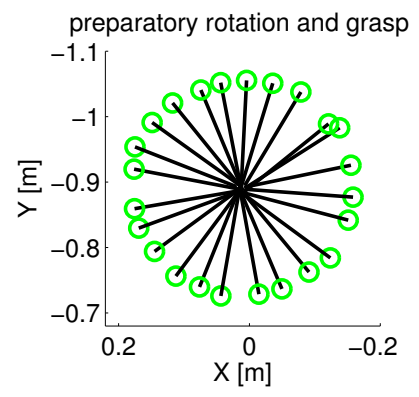

b
Fig. 12. Empirical test results for two open-loop manipulation strategies for transporting the pan. The plotted handle directions are coded according to whether the trial resulted in a successful transport of the pan to the goa position. (a) Initial object pose for the trials using only the grasp action. The grasp sequence can grasp the pan from 4 of 24 tested angles for a 45 degree capture region. (b) Initial object pose for trials using the preparatory rotation action before grasping. The manipulator was able to grasp the pan from all 24 tested angles using rotation.

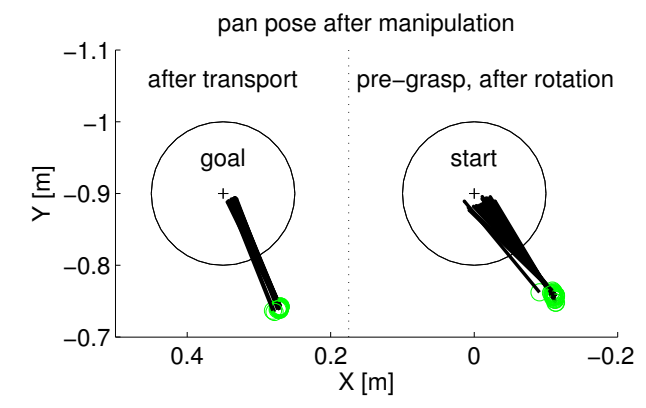

Fig. 13. The object handle pose for the trials using preparatory rotation strategy. After the rotation action (right), the preparatory action has significantly reduced the uncertainty in object pose by pushing the handle into the grasping capture region (15 degree range in orientation). After the grasping action (left), the object has been transported consistently to the final goal position (6 degree range in orientation).

outstretched fingers in the grasp component.

In contrast, when the preparatory rotation action is used prior to the grasping action, the manipulator completed the transport task successfully for all 24 of the 24 consecutive trials (Fig. 12b.) In all but one of the trials, the index finger made contact with the handle at some point during the rotation arc path and pushed the handle clockwise. The one exception was the second leftmost handle angle of the four orientations already within the capture region of the grasping action alone. Because the handle orientation was already centered in the original grasping capture region, the object remained stationary during the rotation action, as intended by the design of the circular push path.

The preparatory rotation action consistently rotated the pan into the grasping action's capture region (Fig. 13). The handle angles after rotation and before the grasp were all within a 15-degree range. The grasping action further reduced the uncertainty in object pose at the goal position. The handle angles after the grasping and transport action were all within a 6-degree range.

\section{Kinematic analysis of alternative grasp reuse strategy}

In our implementation we have focused on the idea of reusing an entire grasp action consisting of the approach motion, grasping motion, and transport motion components for both the arm and the hand. Each motion component was manually programmed for the specific task tested in our experiments. The grasping motion is the most critical component that is manually-programmed, because the hand pose must be carefully tuned in order to securely grasp the thin handle of the pan. During the grasping motion component, the manipulator arm configuration was stationary while the finger joints closed around the pan handle.

An alternative scheme to reuse a well-tuned grasp would be to re-plan the arm configuration and arm motion components without changing the hand motion during the grasp. In this way, as long as the same relative configuration is maintained between the palm of the hand and the object, the same tuned finger motion for grasping can be used with new arm configurations.

We investigated this alternative scheme of reusing the hand motion while re-planning the arm motion using kinematic analysis. For each possible handle orientation of the pan given the same center position, we computed the required palm transform in the workspace required to maintain the same relative configuration to the object handle. Given the desired palm transform, we searched for an inverse kinematics solution of an arm configuration which would achieve the desired end effector (palm) configuration. The inverse kinematics solution was computed iteratively using a pseudo-inverse Jacobian method [16]. Because the inverse kinematics solutions are highly-dependent on the initial guess for the iterative search, multiple initial configurations were tested for each desired palm pose. The guesses were selected from a database of precomputed arm configurations discretized in joint space. Any pre-computed configuration whose end-effector position was in the neighborhood of the desired palm position was evaluated as an initial guess.

The results of the kinematic analysis (Fig. 14) show that the same relative transform between the object and hand is reachable for a wide range of handle orientations much larger than the empirical capture region of the single grasping action. However, about one-third of the possible handle orientations are still unreachable by the manipulator. Thus, even under the considered alternative grasping scheme, preparatory rotation could still be used to achieve successful grasps for unreachable handle directions.

\section{DiscusSion}

Our implementation of a specific open-loop rotation was intended to test the merit of the preparatory rotation strategy in terms of extending the effective grasp capture region. Under this scheme, the pan may be rotated in almost a full circle for some initial configurations. The length of the rotation for some 


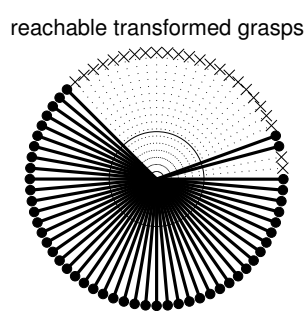

Fig. 14. Reachable object handle orientations computed for a scheme where the reaching motion is re-planned but the relative configuration between hand and object during grasping is the same. If a novel reaching motion is planned for each possible handle orientation, the manipulator may be able grasp from a wider capture region then the single open-loop grasping action. However, about one-third of the orientations are still unreachable.

of the handle directions facing toward the manipulator makes the strategy sensitive to the initial position of the pan. It is possible for the hand to lose contact with the handle during the rotation if the pivot point is not placed properly. The strategy we implemented can be extended by adding additional openloop actions tuned for different sets of initial conditions, which might be determined by only a few bits of sensor data. A simple example would be to minimize the overall pan rotation by using a counterclockwise preparatory motion for handle orientations on the left. This would avoid the need to maintain contact for a long duration along the circular push-path and may improve the robustness of the rotation action in achieving the desired handle configuration prior to the grasp.

In addition to extending the capture region of a welltuned grasping action, the preparatory rotation strategy may be desirable in humanoid robots by making the manipulator motion appear more human-like. Features such as bidirectional rotation and a more relaxed hand pose for multi-finger pushing contact could improve the manipulator's natural appearance.

Other similar preparatory manipulation strategies include sliding, rolling, or tumbling maneuvers which re-configure the object prior to grasping. In the human subject experiments, translational sliding of the object was not constrained in any of the task scenarios. The human motion capture data does reveal that some of the unimanual manipulation resulted in both planar rotation and translation prior to lifting. In the robot experiments, we found that a simple pivoting motion was sufficient to increase the capture region without specifically programming a translational displacement action.

Directions for future work in robotics include examining which features of these preparatory manipulation strategies should be imitated in humanoid robots. Some features, such as the arm configuration, might be essential for a robot to appear human-like. Other features, such as optimization of the joint torques or grasp quality, might be important heuristics for achieving robust performance in difficult task conditions.
Further studies of human motor control may also uncover new concepts that suggest how to improve the robustness of robotic manipulation and anthropomorphism in humanoid robots.

\section{ACKNOWLEDGMENT}

This work was supported by the National Science Foundation (IIS-0326322, ECS-0325383, and CCF-0702443). L. Y. Chang received support from a National Science Foundation Graduate Research Fellowship and a NASA Harriet G. Jenkins Pre-Doctoral Fellowship. The authors thank Howard Seltman for his guidance on the statistical analysis and Justin Macey for his assistance with the data acquisition.

\section{REFERENCES}

[1] M. T. Mason, "Mechanics and planning of manipulator pushing operations," International Journal of Robotics Research, vol. 5, no. 3, pp. 53-71, 1986

[2] K. M. Lynch and M. T. Mason, "Controllability of pushing," in IEEE International Conference on Robotics and Automation, Nagoya, Japan, May 1995, pp. 112-119.

[3] M. Stilman and J. Kuffner, "Navigation Among Movable Obstacles: Real-Time Reasoning In Complex Environments," International Journal of Humanoid Robotics, vol. 2, no. 4, pp. 479-503, 2005.

[4] K. Hauser, V. Ng-Thow-Hing, and H. Gonzalez-Banos, "Multi-modal motion planning for a humanoid manipulation task." in Proceedings of the International Symposium on Robotics Research (ISRR), 2007.

[5] V. Ng-Thow-Hing, E. Drumwright, K. Hauser, Q. Wu, and J. Wormer, "Expanding task functionality in established humanoid robots," in IEEE/RAS International Conference on Humanoid Robots (Humanoids 2007), 2007.

[6] T. Lozano-Perez, M. Mason, and R. H. Taylor, "Automatic synthesis of fine-motion strategies for robots," International Journal of Robotics Research, vol. 3, no. 1, pp. 3-24, 1984.

[7] D. A. Rosenbaum, F. Marchak, H. J. Barnes, J. Vaughan, J. D. Slotta, and M. J. Jorgensen, Attention and Performance XIII: Motor Representation and Control. Hillsdale, NJ: Lawrence Erlbaum Associates, 1990, ch. Constraints for Action Selection: Overhand Versus Underhand Grips, pp. 321-342.

[8] D. A. Rosenbaum, J. Vaughan, M. J. Jorgensen, H. J. Barnes, and E. Stewart, Attention and performance XIV - A silver jubilee: Synergies in experimental psychology, artificial intelligence and cognitive neuroscience. Cambridge: MIT Press, Bradford Books, 1993, ch. Plans for object manipulation, pp. 803-820.

[9] M. W. Short and J. H. Cauraugh, "Planning macroscopic aspects of manual control: end-state comfort and point-of-change effects." Acta Psychologica, vol. 96, no. 1-2, pp. 133-147, Jun 1997.

[10] W. Zhang and D. A. Rosenbaum, "Planning for manual positioning: the end-state comfort effect for manual abduction-adduction." Experimental Brain Research, vol. 184, no. 3, pp. 383-389, Jan 2008

[11] D. A. Rosenbaum and M. J. Gaydos, "A method for obtaining psychophysical estimates of movement costs." Journal of Motor Behavior, vol. 40, no. 1, pp. 11-17, Jan 2008 .

[12] M. K. Rand and G. E. Stelmach, "Effect of orienting the finger opposition space in the control of reach-to-grasp movements." Journal of Motor Behavior, vol. 37, no. 1, pp. 65-78, Jan 2005.

[13] L. Y. Chang and N. S. Pollard, "On preparatory object rotation to adjust handle orientation for grasping," Robotics Institute, Carnegie Mellon University, Pittsburgh, PA, Tech. Rep. CMU-RI-TR-08-10, April 2008.

[14] C. E. Clauser, J. T. McConville, and J. Young, "Weight, volume and center of mass of segments of the human body," Aerospace Medical Research Laboratory, Wright-Patterson Air Force Base, Ohio., Antioch College, Yellow Springs, OH., Tech. Rep. AMRL-TR-69-70, August 1969.

[15] G. Verbeke and G. Molenberghs, Linear mixed models for longitudinal data. New York: Springer, 2000.

[16] P. Corke, "A robotics toolbox for MATLAB," IEEE Robotics and Automation Magazine, vol. 3, no. 1, pp. 24-32, March 1996. 